\title{
In viaggio tra le lingue e le culture
}

\section{Martine Noussan}

\section{(2) OpenEdition}

\section{Journals}

\section{Edizione digitale}

URL: http://journals.openedition.org/esp/1151

DOI: 10.4000/esp. 1151

ISSN: 2532-0319

\section{Editore}

Centre d'Information sur l'Éducation Bilingue et Plurilingue

\section{Edizione cartacea}

Data di pubblicazione: 1 giugno 2017

Paginazione: 87-92

ISSN: 1127-266X

\section{Notizia bibliografica digitale}

Martine Noussan, «In viaggio tra le lingue e le culture », Éducation et sociétés plurilingues [Online], 42

2017, Messo online il 01 février 2018, consultato il 15 septembre 2020. URL : http://

journals.openedition.org/esp/1151 


\title{
IN VIAGGIO TRA LE LINGUE E LE CULTURE
}

\begin{abstract}
Martine NousSAN
Un jour, en fouillant parmi de vieux objets, j’ai trouvé une cassette audio dans laquelle était enregistrée une conversation en patois entre mon frère et moi. Nous étions sans doute très jeunes car je n'en gardais aucun souvenir. Le fait de ne pas reconnaître sa voix enregistrée est une sensation assez courante mais cette fois-là l'extranéité perçue était accentuée par le fait que je ne m’identifiais pas en tant que "parlant" de cette langue, le patois. Autant que je me souvienne, mes grand-parents maternels et mes tantes ont toujours parlé en patois et j'ai toujours tout compris sans difficulté mais je n'ai jamais utilisé activement cette langue.
\end{abstract}

Mots-clés: conversation, patois, grand-parents, extranéité perçue

One day, while rummaging among old things, I discovered a recorder with a tape of a conversation between my brother and me, in patois. We must have been very small, because I don't remember it at all. Not recognizing one's own, recorded voice is a rather common experience, but this time, the feeling of strangeness was even stronger because I didn't identify as a speaker of that language, patois. As far back as I can remember, my maternal grandparents and aunts always spoke patois and I never had trouble understanding but never used the language actively myself.

Key-zords: conversation, patois, grand-parents, perceived strangeness

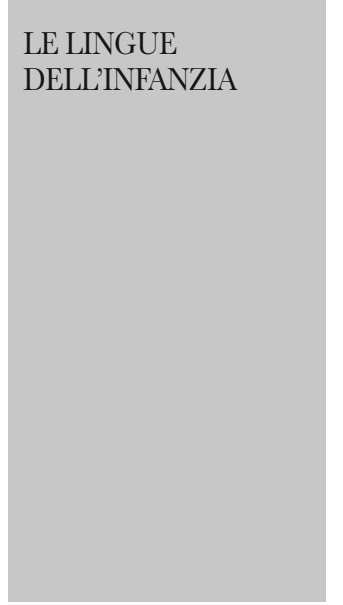

$\mathrm{U}$ n giorno, rovistando tra vecchi oggetti, ho trovato una musicassetta su cui era registrata una conversazione tra me e mio fratello in patois. Dovevamo essere davvero piccoli, perché non ne avevo alcun ricordo. Quella di non riconoscere la propria voce registrata è una sensazione piuttosto comune, ma quella volta l'estraneità percepita era accentuata dal fatto che non mi riconoscessi affatto in quanto "parlante" di quella lingua, il patois. Per quel che ricordo, i miei nonni materni e le mie zie hanno sempre parlato in patois, io ho sempre capito tutto senza alcuna difficoltà, ma non ho mai usato attivamente questa lingua. Quella musicassetta dimostrava invece il contrario, e per me era stata una grande scoperta! Non saprei dire a che età e per quale motivo (forse l'influenza della scuola elementare?) ho smesso di utilizzarlo per passare all'italiano, ma oggi il patois è per me una lingua legata all'infanzia e alla casa dei miei nonni, calda e familiare, che mi riporta alle mie radici. Quando devo pensare a delle 


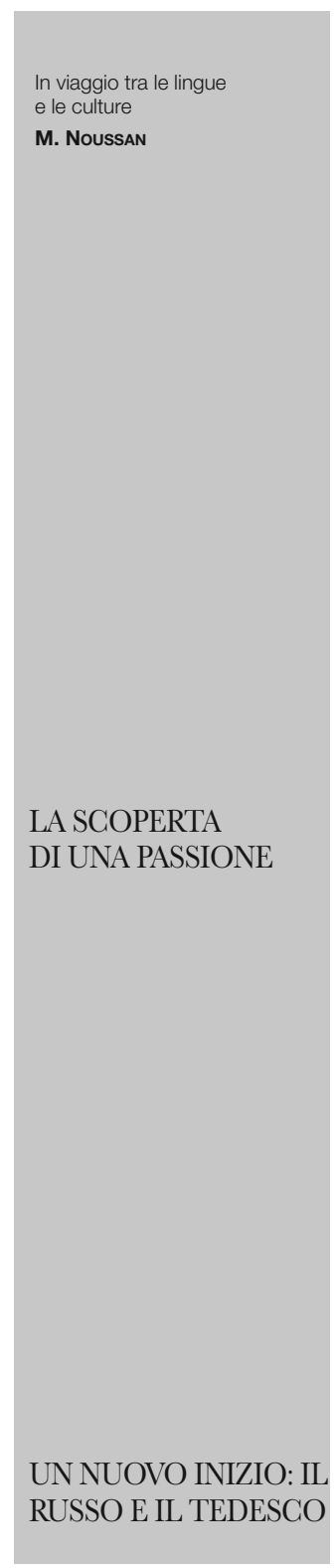

parole in patois ho sempre in mente l'immagine di mia nonna che le pronuncia, mentre dette da me mi sembrerebbero poco naturali.

Ai nonni paterni devo forse invece il primo contatto con il francese, ancora adesso con mia nonna parlo in questa lingua e ancora adesso io e mio fratello a volte ci divertiamo a farla arrabbiare usando delle espressioni inventate a partire dall'italiano come "ça capite" invece di "ça arrive". Il francese è comunque una materia in cui non mi sono mai davvero applicata, forse anche a causa di una professoressa delle superiori che aveva un'enorme cultura (sia linguistica sia generale), ma nessuna capacità di trasmetterla agli studenti. Diciamo che è una lingua che mi sembra innata, in Valle d'Aosta la studiamo a scuola per 13 anni, quindi volenti o nolenti ci entra nelle orecchie.

Delle scuole elementari più che lo studio mi ricordo gli intervalli di mezza mattinata passati a giocare in cortile e le scorribande pomeridiane in bicicletta con i compagni, non saprei quindi dire niente riguardo al mio rapporto con le lingue in quell'epoca, e la stessa cosa vale per le scuole medie, di cui invece mi ricordo soprattutto la fatica fatta per rimediare alle lacune delle elementari.

È alle superiori che, pur avendo scelto un liceo scentifico, mi rendo conto che i miei punti di forza emergono proprio nelle materie umanistiche, nelle lingue e letterature. In italiano non ho mai avuto problemi e i "temi" erano i miei compiti in classe preferiti, per merito di una professoressa molto brava e anche di mia madre, che fin da quando ero piccola è riuscita a trasmettermi il piacere della lettura. Ed è sempre qui che un'altra professoressa molto brava risveglia il mio interesse per l'inglese, lingua che mi apre il mondo della musica, di internet e dei viaggi. Passo infatti delle ore in internet a tradurre e imparare $\mathrm{i}$ testi in inglese delle mie canzoni preferite, e d'estate partecipo a vacanze studio in Inghilterra e Irlanda: ricordo ancora la libertà respirata in quei soggiorni, che erano i miei primi viaggi da sola con le amiche, e la gioia che provavamo nel buttarci a capofitto in ogni avventura. Di sicuro queste esperienze hanno dato una forte spinta alla mia voglia di studiare l'inglese, che purtroppo però è poi finito nel dimenticatoio per molti anni.

All'università infatti, dopo alcune titubanze, scelgo di iscrivermi alla facoltà di lingue, e, guidata dal bisogno di fare qualcosa di completamente nuovo e di ripartire da zero, scelgo il russo e il 


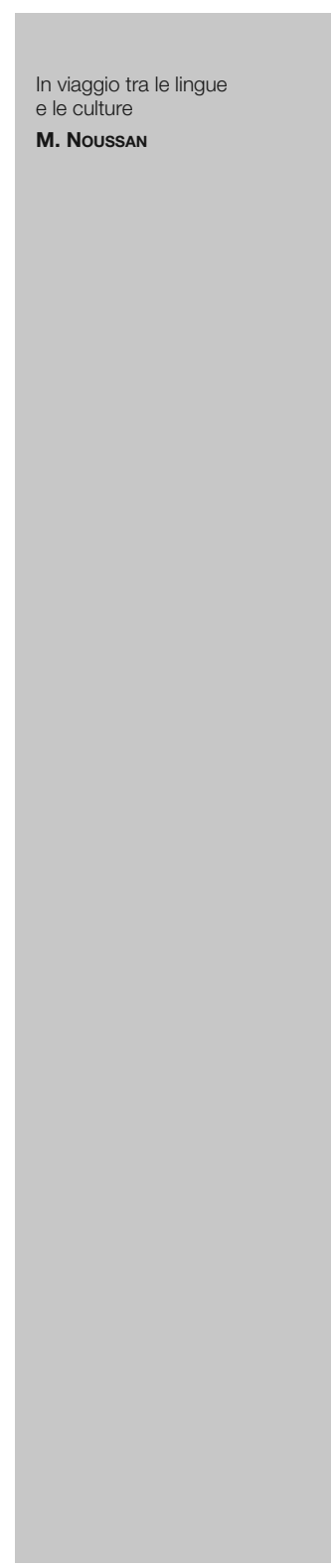

tedesco. Perché proprio queste due lingue? È una domanda che mi fanno in molti, e a cui non so dare una risposta molto precisa. Ho sempre avuto il sogno di fare la transiberiana e dopo aver letto alcuni romanzi di Dostoevskij alle superiori ho provato una forte attrazione verso quell'universo che è "l'uomo russo". Annoiata dal francese e dall'inglese, mi rivolgo alle altre maggiori lingue europee e scelgo il tedesco un po' per caso (lo spagnolo mi sembra troppo simile all'italiano e quindi poco attraente, oltretutto l'avevo già studiato un po' per conto mio alle superiori). Ed è così che mi ritrovo all'Università di Torino, che susciterà in me momenti di entusiamo estremo, ma a volte anche di sconforto. Ricordo gli anni della laurea triennale con enorme piacere e nostalgia, sono anni in cui ho imparato a essere più indipendente, ho coltivato salde amicizie che nonostante le difficoltà logistiche durano tuttora e, soprattutto, ho tratto enormi soddisfazioni dallo studio di queste due lingue: il contatto continuo con la cultura russa e tedesca hanno contribuito non poco a fare di me la persona che sono oggi. Più che gli esami universitari (alcuni utili, altri ridicoli, e qui stendo un velo pietoso sulle condizioni in cui versa l'università italiana), a essere davvero formative per le mie competenze linguistiche sono state le esperienze all'estero che ho fatto in quegli anni.

La prima, due mesi di studio a Mosca, ancora oggi rimane uno dei periodi più felici e spensierati della mia vita. E stata un'immersione totale in un mondo lontano, fino ad allora solo immaginato attraverso la meravigliosa lente della letteratura russa. Insieme a due compagne di viaggio perfette, ho esplorato la capitale in lungo e in largo, alla ricerca di case di scrittori, di luoghi in cui erano stati o di cui avevano narrato, permeati dal peso della storia; alla ricerca dei tratti e abitudini dell'uomo russo che avevamo così tanto amato nella letteratura. Ed è qui che sboccia del tutto l'amore per il russo, questa lingua così "estranea" rispetto alle nostre lingue europee, praticamente incomprensibile all'inizio del nostro soggiorno, ma che progressivamente diventa sempre più familiare. Le condizioni per migliorare le competenze linguistiche c'erano tutte: un mondo nuovo da scoprire, delle amiche e compagne di studio curiose e determinate, dei corsi all'università interessanti. $\mathrm{Al}$ termine del soggiorno mi sembrava di aver imparato di più in quei due mesi a Mosca che nei due anni precedenti all'università di Torino, e l'amore e la spinta che da allora provo verso questa città hanno contribuito a mantenere viva la mia voglia di imparare questa lingua. Un anno dopo, la mia prima esperienza in Germania: un mese a Francoforte, troppo poco per 


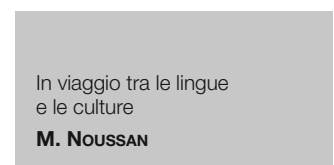

METTERSI IN GIOCO

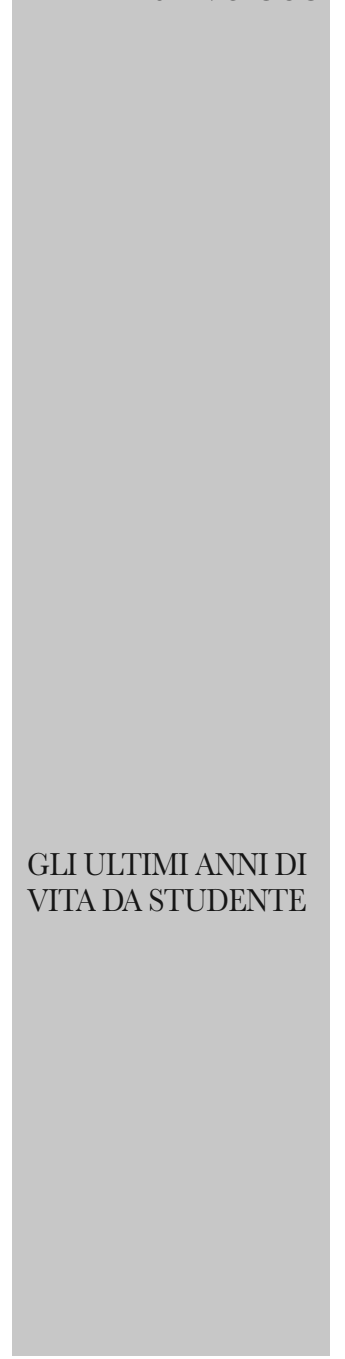

notare un vero progresso nelle mie conoscenze linguistiche (anche perché questa volta sono in Europa, e spesso le persone iniziano a parlarmi in inglese appena si accorgono che non parlo bene tedesco).

Mi laureo a febbraio, e mi sembra di non avere la padronanza di nessuna delle due lingue, ma so che ho ancora tempo di imparare. Nell'attesa di iscrivermi alla laurea magistrale cerco disperatamente di fare un'esperienza all'estero di qualche mese, e finalmente trovo l'occasione giusta: a maggio parto per l'Uzbekistan in qualità di accompagnatrice turistica, e continuo poi la mia esperienza a Mosca e San Pietroburgo. Oltre a vedere posti meravigliosi, ho avuto modo di venire a contatto con la complessità e le contraddizioni del mondo russo, ho ampliato le mie competenze linguistiche (sia nell'ambito della quotidianità, sia in quello più specifico delle formalità doganali e dei veicoli, lavoravo infatti con turisti in camper) e soprattutto ho imparato a sfruttare risorse che neanche credevo di avere. Questo lavoro mi ha infatti costretto a superare la mia timidezza, mi ha insegnato ad arrangiarmi nei numerosi momenti di difficoltà (causati dalla disorganizzazione dell'agenzia per cui lavoravo e dalla burocrazia russa), e obbligandomi a interagire il più possibile con l'ambiente circostante per far fronte a vari problemi mi ha permesso di acquisire una notevole dimestichezza con il russo. E durante questa esperienza che, avendo a che fare con turisti francesi, ho rivalutato questa lingua e mi sono resa conto della fortuna che ho ad averla studiata, anche se non la praticavo davvero ormai da un paio d'anni l'ho infatti ripresa velocemente e senza alcuna difficoltà.

Mi iscrivo poi alla laura magistrale a Bologna e mi butto a capofitto nello studio, col russo mi sento molto più sicura di prima, col tedesco faccio ancora fatica, ma comunque lo studio ripaga e mi dà moltissime soddisfazioni. Il secondo anno parto per Dresda con il progetto Erasmus, un po' spaventata per via del tedesco, lingua in cui non mi sento ancora mio agio, un po' svogliata perché avrei preferito andare in Russia. E invece si rivela essere uno degli anni più belli della mia vita. La vita lì mi sembra facile e spensierata, mi sento priva di qualunque responsabilità, libera e ben accolta dalla società tedesca. Anche qui trovo due fantastiche amiche con cui condivido le gioie e le difficoltà della lingua tedesca, le lezioni, gli esami, gli incontri di tandem linguistico con altri ragazzi, le nuove amicizie. Durante quest'anno, tramite l'università, ho anche partecipato a un seminario a Ekaterinburg, in 


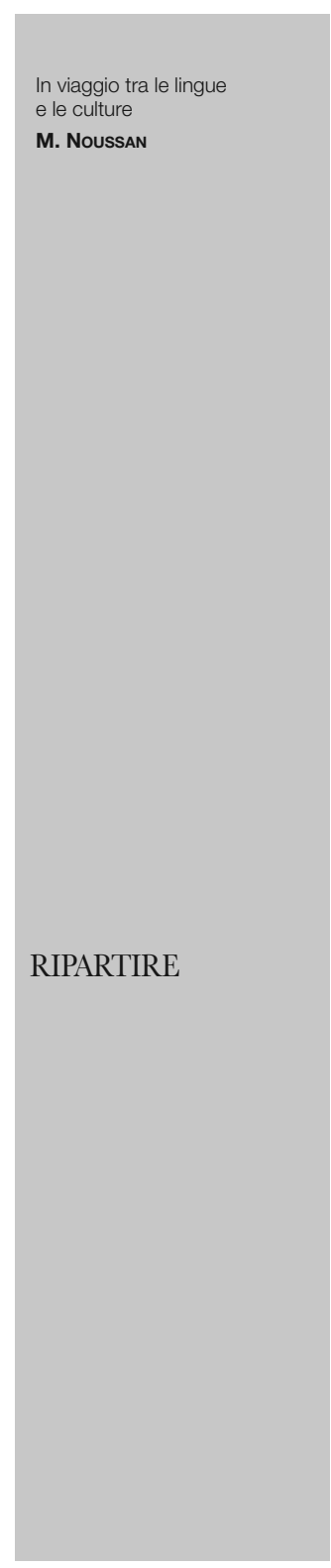

Russia. È stata un'esperienza molto particolare, perché per le mie compagne di viaggio tedesche la lingua straniera su cui concentrarsi era in quel caso il russo, mentre per me la difficoltà era doppia: passare continuamente dal russo al tedesco e viceversa richiedeva uno sforzo non indifferente, e portava inevitabilmente a vari miscugli linguistici, soprattutto con espressioni brevi che mi risultavano più "automatiche", per esempio, quante volte ho scambiato "ja" e "da" "ja" in tedesco vuol dire "si", in russo "io", "da" in russo vuol dire "si", in tedesco "qui, li")! Sebbene io avessi già fatto esperienze all'estero, non sono mai stata così male come al momento di dover lasciare Dresda, è stata la prima volta in cui mi è sembrato davvero impossibile tornare alla mia vecchia vita italiana. Eppure così è stato, e per fortuna ho avuto il piacere di riscoprire Torino, da cui mancavo ormai da tre anni, che mi ha dato la tranquillità necessaria per trovare la forza di scrivere una tesi in traduzione e finire così gli studi.

Uno dei motivi per cui avevo scelto di iscrivermi alla facoltà di lingue era che mi sembrava che le lingue mi avrebbero aperto porte infinite. Dopo la laurea mi sembra invece che in realtà non ne aprano neanche una. Mi sembra di aver studiato delle materie interessantissime, ma di non aver acquisito le competenze necessarie a usare le lingue a un livello professionale, e cerco quindi di mettermi in testa il solito pensiero: non finirò mai di imparare, quindi non mi sentirò mai davvero sicura in una lingua, ed è normale che sia così.

Dopo un annetto di viaggi e riposo (e un'esperienza di traduzione di due documentari per il sottotitolaggio che ha messo alla prova il mio tedesco) trovo un'altra occasione per partire. Riesco finalmente a coronare uno dei miei sogni, e faccio un tratto di transiberiana per arrivare fino all'isola di Olkhon, sul lago Bajkal, dove lavoro per tre mesi in un ostello. È un'esperienza ancora "fresca", e non ho quindi la giusta distanza per riuscire a considerarla con una certa oggettività. Mi sono sentita davvero viva su quell'isola persa in mezzo al nulla, ogni giorno è stato un'avventura, ho svolto le mansioni più svariate, dall'accoglienza dei turisti alla reception, all'aiuto cuoca, al lavoro in dispensa, alle lezioni di conversazione in francese, all'accompagnatrice turistica nelle escursioni, sono entrata in contatto con tantissime persone, molte delle quali davvero speciali. Per quanto riguarda il russo non mi sembra di aver fatto progressi enormi, mi trovavo in un ambiente piuttosto internazionale, e quindi diciamo che ho fatto più che altro un grande ripasso di tutte le lingue che conosco, italiano e russo, ma anche 


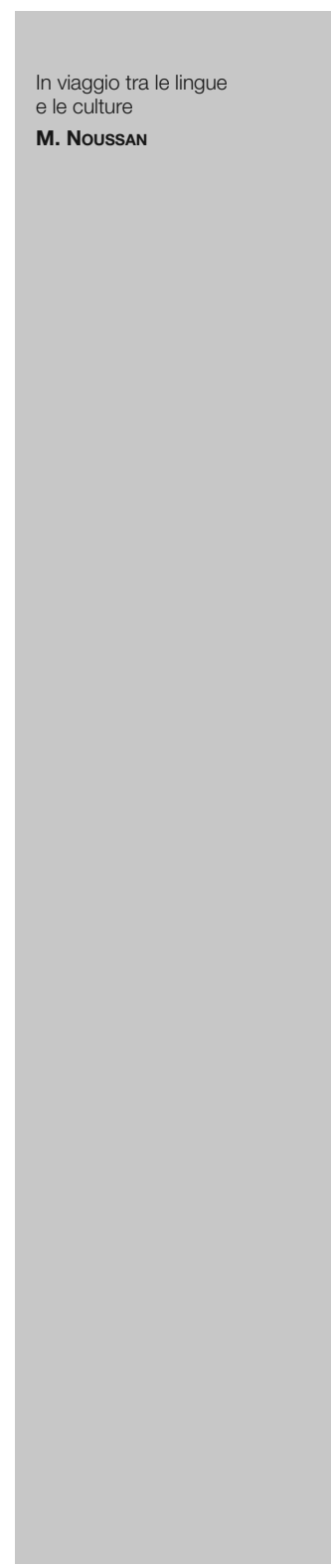

francese e tedesco, e soprattutto, dopo ben otto anni, ho riniziato a parlare inglese. E questa, dopo Dresda, è stata la seconda volta in cui ho lasciato un luogo con le lacrime agli occhi e un groppo in gola. Da quest'esperienza però ho capito che le lingue aprono davvero molte strade, se si è pronti ad accettare certe condizioni.

Un mese dopo il lago Bajkal mi sono di nuovo spostata: ora mi trovo a Mainz, in Germania, dove lavorerò come assistente di italiano in un liceo fino alla fine di maggio. È la mia prima esperienza da insegnante, e sono curiosa di capire se è un lavoro che mi piace e che fa per me.

Sento che la mia vita in questi ultimi anni ha sempre oscillato tra due poli, ovvero le due lingue che ho studiato, il russo e il tedesco. Continuo a stupirmi di come possano piacermi due paesi così diversi e spesso opposti tra loro: la Germania è pulita, efficiente, attenta all'ambiente, è facile e comodo vivere qui, forse troppo. La Russia (purtroppo) è spesso sporca, inquinata, caotica, per viverci devi saperti arrangiare, e forse è questo che la rende così stimolante (mi rendo conto che questa è una forte semplificazione, ma se dovessi davvero descrivere questi due paesi mi servirebbe un libro intero). Nella dicotomia tra sentimento e ragione direi che per me la Russia sta dalla parte del sentimento, e la Germania della ragione, cosa che scaturisce in parte anche dalle lingue: il tedesco è preciso e analitico, e possiede strutture rigide, il russo invece grazie alla presenza dei casi può costruire le frasi in maniera estremamente libera, e per esempio l'utilizzo degli aspetti verbali dipende per la maggior parte dal contesto. Sono davvero felice di aver potuto assimilare queste due strutture mentali così differenti, e mi sento davvero fortunata ad aver scelto quasi per caso due lingue così affascinanti che mi hanno portato, e spero continuino a portarmi, a fare delle esperienze importanti. 